\title{
Correction to: Two different therapies for the middle turbinate during endoscopic sinus surgery for chronic rhinosinusitis
}

\author{
Meichan Zhu ${ }^{1}$ (i) $\cdot$ Yongyi Yan ${ }^{1} \cdot$ Huicheng Gong ${ }^{1} \cdot$ Yunwen Wu ${ }^{1} \cdot$ Guojie $\operatorname{Tan}^{1}$
}

Published online: 1 August 2020

○) Springer-Verlag GmbH Germany, part of Springer Nature 2020

\section{Correction to: European Archives of Oto-Rhino-Laryngology \\ https://doi.org/10.1007/s00405-020-06184-4}

In the original publication of the article, Fig. 1a was missing and caption of Fig. 1a was published as caption of Fig. $1 \mathrm{~b}$. The correct Fig. 1 and captions are provided below.

The original article was updated.

$\triangle$ Yongyi Yan yungyi@188.com

1 Department of Otorhinolaryngology, Guangzhou Twelfth People's Hospital (Guangzhou Otolarynology-Head and Neck Surgery Hospital), Tianhe District, No 1.

Tianqiang Road, Guangzhou 510620, Guangdong, China 
a

\begin{tabular}{|c|c|c|c|}
\hline Middle turbinate & score & Left & Right \\
\hline Normal & 0 & & \\
\hline Synechia/lateralized & $1-2$ & & \\
\hline \multicolumn{4}{|l|}{ Middle } \\
\hline \multicolumn{4}{|l|}{ Meatus/MMA } \\
\hline Healthy & 0 & & \\
\hline Narrowing/Closure & $1-2$ & & \\
\hline $\begin{array}{l}\text { Maxillary sinus } \\
\text { contents }\end{array}$ & $1-2$ & & \\
\hline \multicolumn{4}{|l|}{ Ethmoid Cavity } \\
\hline Healthy & 0 & & \\
\hline Crusting & $1-2$ & & \\
\hline Mucosal Edema & $1-2$ & & \\
\hline Polypoid Change & $1-2$ & & \\
\hline Polyposis & $1-2$ & & \\
\hline Secretions & $1-2$ & & \\
\hline \multicolumn{4}{|l|}{ Secondary Sinuses } \\
\hline Frontal Recess/Sinus & $0-2$ & & \\
\hline Sphenoid Sinus & $0-2$ & & \\
\hline \multirow[t]{2}{*}{ Total Score } & 16 & $18 \mathrm{~F}$ & \\
\hline & $18 \mathrm{~S}$ & 20 & \\
\hline
\end{tabular}

Fig. 1 A POSE scoring system. The scoring criteria for POSE were as follows: synechia to the lateral wall or lateralized middle turbinate $=1$ point each; narrow middle meatus $=1$ or closed middle meatus $=2$; maxillary sinus with mucoid secretions/edema $=1$ or with purulence/allergic mucin $=2$; ethmoid cavity with few isolated crusts $=1$ or with diffuse or occluding crusting $=2$; ethmoid cavity with mucosal edema (loss of discernible underlying bony contours in some areas) $=1$ or with mucosal edema (diffuse loss of discernible underlying bony contours $)=2$; ethmoid cavity with polypoid changes partially filling the cavity $=1$ or entirely filling the cavity $=2$; ethmoid cavity with polyposis beyond the middle meatus but not to the inferior turbinate $=1$ or beyond the upper border of the inferior turbinate $=2$; ethmoid cavity with thin/mucoid secretions $=1$ or with purulent/allergic mucin $=2$; narrowed frontal recess/ sinus or edema present in the recess/sinus $=1$ or obstructed/infected/ severely inflamed frontal recess/sinus $=2$; and narrowed sphe- noid sinus or edema present in the sinus $=1$ or obstructed/infected/ severely inflamed sphenoid sinus $=2$. The total scores were based on the extent of surgery: $16=$ total score (middle meatal antrostomy + ethmoidectomy); $18 \mathrm{~F}=$ total score (middle meatal antrostomy + ethmoidectomy + frontal sinusotomy); $18 \mathrm{~S}=$ total score (middle meatal antrostomy + ethmoidectomy + sphenoidotomy); $20=$ total score (middle meatal antrostomy + ethmoidectomy + sphenoidotomy + frontal sinusotomy). MMA = middle meatal antrostomy; $\mathrm{POSE}=$ perioperative sinus endoscopy. $\mathbf{B}$ Middle turbinate treatment. a Injection saline of middle turbinate. $b$ Incision anterior middle turbinate. $c$ Incision inferior middle turbinate. $d$ Separation of the bone of middle turbinate. e Take out the bone of middle turbinate. $f$ Take out broken bone of middle turbinate. $\mathrm{g}$ Take out remainder of bone middle turbinate. $\mathrm{h}$ Restoration of middle turbinate. i Packing gelatin sponge between the middle turbinate and nasal septum. $\mathrm{j}$ Packing gelatin sponge between the lateral nasal wall and middle turbinate 
b

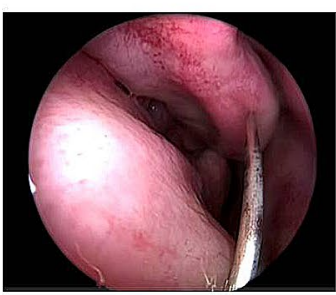

A

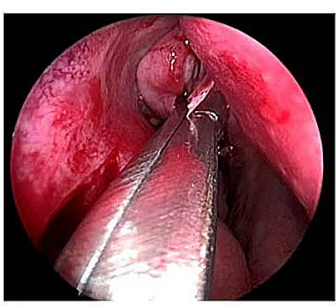

F

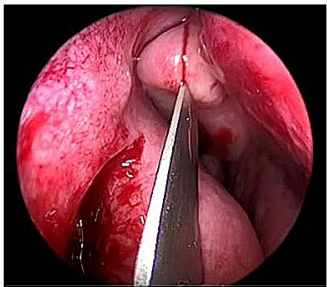

B

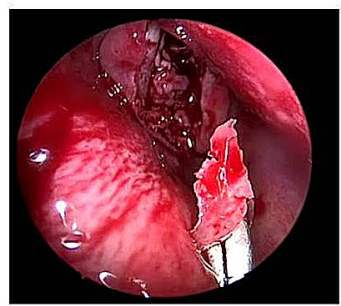

G

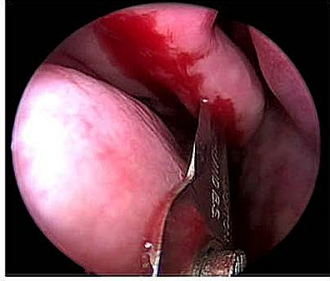

C

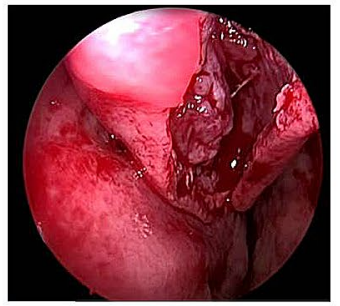

$\mathrm{H}$

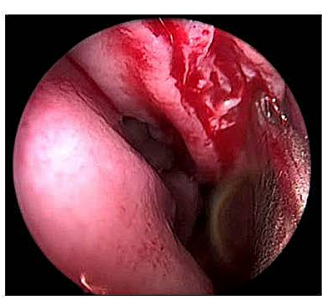

D

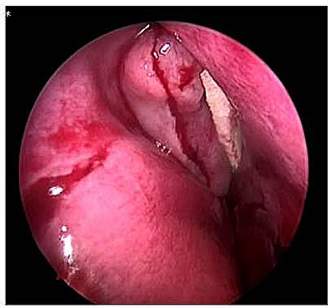

I

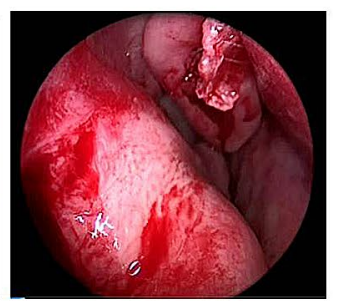

E

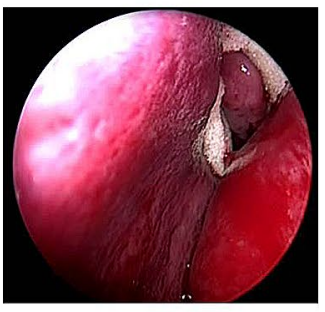

J

Fig. 1 (continued)

Publisher's Note Springer Nature remains neutral with regard to jurisdictional claims in published maps and institutional affiliations. 